Proc. of the 15th Int. Workshop on Slow Positron Beam Techniques and Applications, Prague, September 2-6, 2019

\title{
Accumulation of Positrons from a LINAC Based Source
}

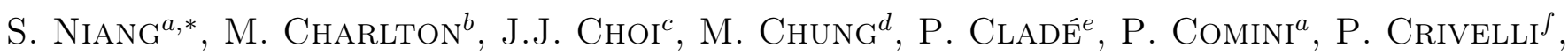

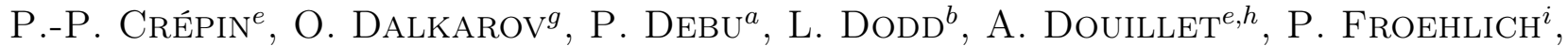
J. Gafriller ${ }^{j}$, S. Guellati ${ }^{e}$, J. Heinrich ${ }^{e}$, P.-A. Hervieux ${ }^{k}$, L. Hilico $^{e} h$, A. Husson ${ }^{l}$,

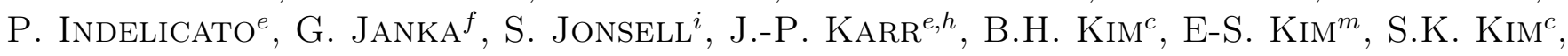
A. Kleyheeg ${ }^{j}, \mathrm{Y} . \mathrm{Ko}^{n}, \mathrm{~T} \cdot \mathrm{Kosinski}^{o}, \mathrm{~N} \cdot \mathrm{Kuroda}^{p}, \mathrm{~B} \cdot \mathrm{LAtacz}^{a}, \mathrm{H} \cdot \mathrm{LeE}^{c}, \mathrm{~J}_{\mathrm{LeE}}{ }^{n}$, A.M.M. Leite ${ }^{a}$, E. Lim $^{m}$, L. Liszkay ${ }^{a}$, T. Louvradoux ${ }^{e}$, D. Lunney ${ }^{l}$, K. LÉvéque $^{k}$, G. Manfredi ${ }^{k}$, B. Mansoulié ${ }^{a}$, M. Matusiak ${ }^{o}$, G. Mornacchi $^{j}$, V.V. Nesvizhevsky $^{q}$,

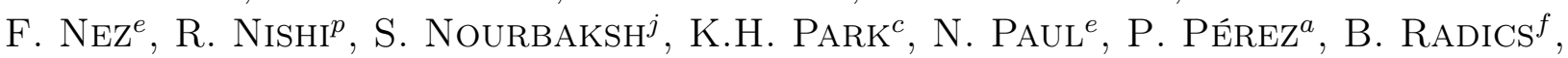

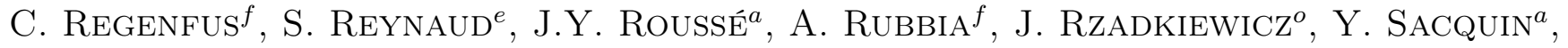
F. Schmidt-Kaler ${ }^{r}$, M. Staszczak ${ }^{o}$, B. Tuchming ${ }^{a}$, B. VAllage $^{a}$, D.P. VAn der Werf ${ }^{b}$,

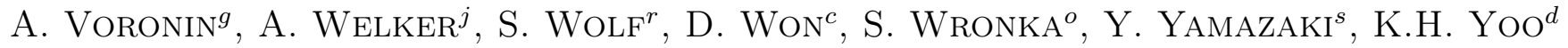
(GBAR COLlaboration) AND C.J. BAKER ${ }^{b}$

${ }^{a}$ IRFU, CEA, Université Paris-Saclay, F-91191 Gif-sur-Yvette, France

${ }^{b}$ Department of Physics, College of Science, Swansea University, Swansea SA2 8PP, United Kingdom

${ }^{c}$ Department of Physics and Astronomy, Seoul National University, 1 Gwanak-Ro, Gwanak-gu, Seoul 08826, Korea

${ }^{d}$ Department of Physics, Ulsan National Institute of Science and Technology (UNIST), 50, UNIST-gil, Ulsan 44919, Republic of Korea

${ }^{e}$ Laboratoire Kastler Brossel, Sorbonne Université, CNRS, ENS-PSL Research University, Collège de France,

Campus Pierre et Marie Curie, 4, pl. Jussieu, F-75005 Paris, France

${ }^{f}$ Institute for Particle Physics and Astrophysics, ETH Zurich, 8093 Zurich, Switzerland ${ }^{g}$ P.N. Lebedev Physical Institute, 53 Leninsky Prospect, 119991 Moscow, Russia

${ }^{h}$ Université d'Evry-Val d'Essonne, Université Paris-Saclay, Boulevard François Mitterand, F-91000 Evry, France ${ }^{i}$ Department of Physics, Stockholm University, SE-10691 Stockholm, Sweden

${ }^{j}$ CERN, 1211 Geneva 23, Switzerland

${ }^{k}$ Université de Strasbourg, CNRS, Institut de Physique et Chimie des Matériaux de Strasbourg,

UMR 7504, F-67000 Strasbourg, France

${ }^{l}$ CSNSM-IN2P3-CNRS, Université de Paris Sud, Orsay, France

${ }^{m}$ Department of Accelerator Science, Korea University Sejong Campus,

Sejong-ro 2511, 30019 Sejong, Republic of Korea

${ }^{n}$ Center for Underground Physics, Institute for Basic Science,

70 Yuseong-daero 1689-gil, Yuseong-gu, Daejeon 34047, Korea

${ }^{\circ}$ National Centre for Nuclear Research (NCBJ), A. Soltana 7, 05-400 Otwock, Swierk, Poland

${ }^{p}$ Institute of Physics, University of Tokyo, 3-8-1 Komaba, Meguro, Tokyo 153-8902, Japan

${ }^{q}$ Institut Max von Laue - Paul Langevin (ILL), 71 av. des Martyrs, Grenoble, France, F-38042

${ }^{r}$ QUANTUM, Institut für Physik, Johannes Gutenberg Universität, D-55128 Mainz, Germany

${ }^{s}$ Ulmer Fundamental Symmetries Laboratory, RIKEN, 2-1 Hirosawa, Wako, 351-0198, Saitama, Japan

The GBAR experiment aims to measure the gravitational acceleration of antihydrogen $\overline{\mathrm{H}}$. It will use $\overline{\mathrm{H}}^{+}$ions formed by the interaction of antiprotons with a dense positronium cloud, which will require about $10^{10}$ positrons to produce one $\overline{\mathrm{H}}^{+}$. We present the first results on the positron accumulation, reaching $3.8 \pm 0.4 \times 10^{8} e^{+}$collected in $560 \mathrm{~s}$.

DOI: 10.12693/APhysPolA.137.164

PACS/topics: positron plasma, positron accumulation, antimatter, Penning-Malmberg trap, Greaves-Surko trap, GBAR

${ }^{*}$ corresponding author 


\section{Introduction}

The GBAR collaboration aim is to measure the acceleration of antihydrogen atoms in the Earth gravitational field [1]. In the GBAR scheme, one first produces $\overline{\mathrm{H}}^{+}$ ions [2], which are subsequently sympathetically cooled using laser cooled $\mathrm{Be}^{+}$ions [3]. When the anti-ion is cold enough, the extra positron is removed using a laser pulse and the acceleration is derived by timing the subsequent annihilation products of the $\overline{\mathrm{H}}$. The antiprotons are obtained from the ELENA decelerator at CERN [4], while positronium is produced by implantation of about $10^{10}$ positrons into a mesoporous silica film at a kinetic energy of $4 \mathrm{keV}[5]$.

To obtain the necessary positrons, a $9 \mathrm{MeV}$ LINAC accelerates electrons into a tungsten target equipped with a mesh moderator biased at $50 \mathrm{~V}$. The resulting low energy positrons are then accumulated in a buffer gas trap (BGT) and transferred in a high field trap (HFT).

The BGT is a Penning-Malmberg trap based on the Greaves-Surko scheme [6], and was developed at CEA Saclay [7]. It uses $\mathrm{N}_{2}$ as the trapping gas and $\mathrm{CO}_{2}$ as a cooling gas. The trap is constructed from 3 sets of electrodes. In the first stage, inelastic collisions occur under a typical $\mathrm{N}_{2}$ pressure of $10^{-3}$ mbar, whilst the second one is used for the accumulation at a typical $\mathrm{N}_{2}$ pressure of $10^{-4}$ mbar, with the final stage used to store positrons for a dozens of seconds with a typical $\mathrm{N}_{2}$ pressure of $10^{-6}$ mbar. The magnetic field in this trap is $\approx 0.05 \mathrm{~T}$ and an electric potential between $-140 \mathrm{~V}$ and $140 \mathrm{~V}$ can be applied to the electrodes, as appropriate.

The HFT [8] is a $5 \mathrm{~T}$ Penning-Malmberg trap with 27 electrodes each able to hold potentials between $-1 \mathrm{kV}$ and $1 \mathrm{kV}$. The base pressure is lower than $10^{-9}$ mbar. This trap is used for long time storage.

In this article we present the outcome of optimisation studies to trap a large cloud of positrons. The final goal is to accumulate $10^{10}$ positrons in $100 \mathrm{~s}$. The two last previous record are $1.2 \times 10^{9}$ in $2.5 \mathrm{~h}$ [9] and $4 \times 10^{9}$ in $4.5 \mathrm{~h} \mathrm{[10].}$

\section{Studies of the trapping conditions}

After an accumulation time $t$ in the BGT second stage, using the potential profile shown in Fig. 1a, the positron cloud is axially compressed, and then dumped on a target at the exit of the trap (Fig. 1b,c) after the BGT third stage. The positron number is estimated by detecting the $\gamma$ rays resulting from their annihilations on the target using a CsI detector. The signal is proportional to the number of accumulated positrons, which evolves as $N(t)=R \tau\left(1-\mathrm{e}^{-\frac{t}{\tau}}\right)$, where $N$ is the number of positrons, $\tau$ is the lifetime, and $R$ is the trapping rate. Some accumulation curves for $e^{+}$in the second stage are presented in Fig. 2a for different $\mathrm{N}_{2}$ pressures as measured at the injection point of the system. If $t \ll \tau, N(t) \approx R t$, the only parameter to optimise is then $R$. For the rest of the

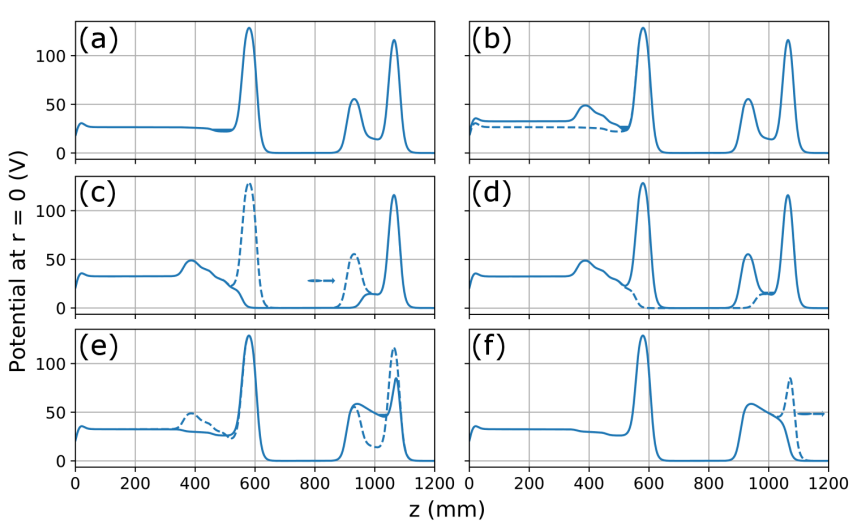

Fig. 1. On-axis potentials used in the buffer gas trap. (a) Accumulation in the second stage. (b) Axial compression of the cloud. (c) Transfer from the second to the third stage. (d) Re-trapping. (e) Preparation for transfer/dump. (f) Dump.
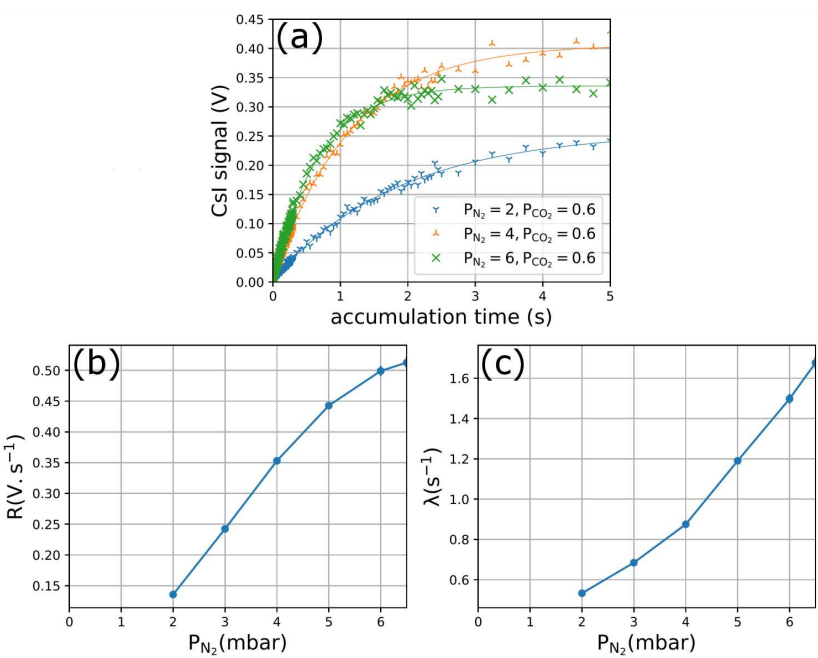

Fig. 2. (a) Number of $e^{+}$as measured by the CsI detector, fit with $N(t)+c$. (b) $R$ vs. $\mathrm{N}_{2}$ pressure. (c) Positron annihilation rate $(\lambda=1 / \tau)$ vs. $\mathrm{N}_{2}$ pressure. Pressures in mbar as measured at the injection point of the system.

experiment we keep $P_{\mathrm{N}_{2}}=6$ mbar, with $\tau=0.67 \pm 0.01 \mathrm{~s}$ and we accumulate for $100 \mathrm{~ms}$ before transferring to the third stage (Fig. 1c,d).

Currently, the energy spread in the longitudinal direction of the incoming positrons is $\sigma=8.8 \pm 0.3 \mathrm{eV}$ but a spread of energy smaller than $3 \mathrm{eV}[6]$ would increase $R$ and therefore the overall efficiency.

In the third stage, the $e^{+}$lifetime is $16.5(0.5) \mathrm{s}$, as shown in Fig. 3a, and since this is much greater than $100 \mathrm{~ms}$ a stacking procedure of the positrons from the second stage can be done. Figure $3 \mathrm{~b}$ shows that, using a static potential, only 6 stacks can be accumulated in the well in a linear way, beyond which the stacks are lost. It also shows that by lowering the bottom of the well 

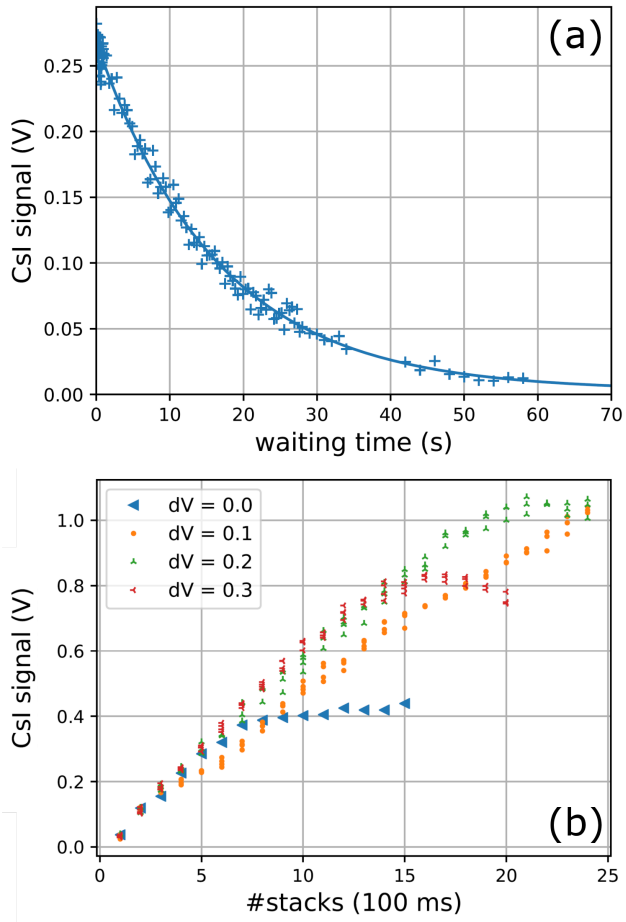

Fig. 3. (a) Lifetime of positrons in the third stage. (b) Measurement of positron signal for different accumulation conditions in stage 3 (see text for details). The bottom of the well is lowered by $\mathrm{d} V$ between each stack.
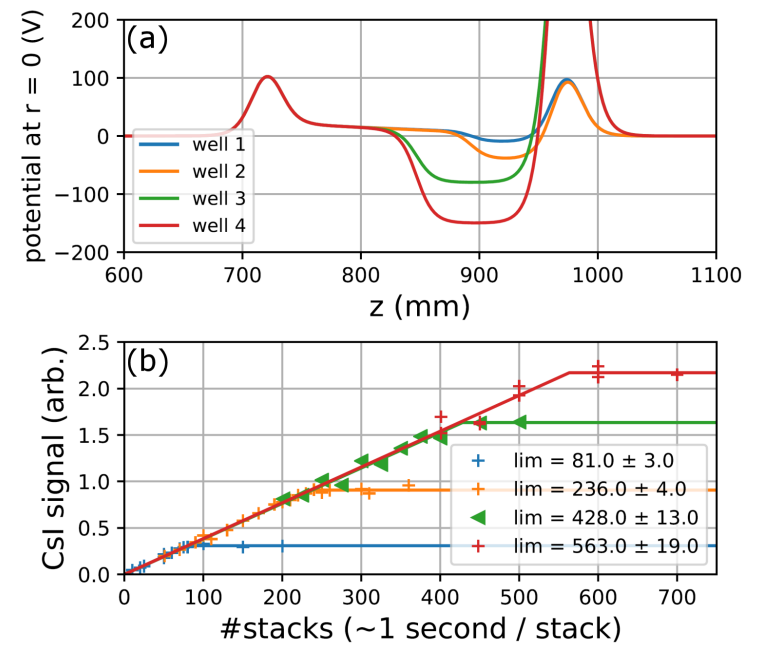

Fig. 4. (a) Subsequent on-axis potentials in the HFT. (b) Accumulation signal from HFT, for respectively a 1-, 2-, 3- and 4-step sequence.

by $0.2 \mathrm{~V}$ per stack, a dozen stacks can be accumulated without any loss. Therefore 10 stacks are accumulated in $1 \mathrm{~s}(10 \times 100 \mathrm{~ms})$ before being transferred to the HFT.

To stack positrons from the BGT third stage in the HFT, one first uses a potential well made of 3 electrodes with $-10 \mathrm{~V}$ as bottom of the well as shown in Fig. 4a.
Figure $4 \mathrm{~b}$ shows that the well is saturated after 80 stacks. To accumulate positrons in a linear way, a sequence is used in which the well is enlarged and deepened each time we approach a saturation limit. In the first well, 50 stacks are accumulated, after which the bottom of the well is lowered. Then 150 stacks are added, and again the bottom of the well is lowered and then further 200 stacks are added. Finally, we lower the bottom of the well to reach a maximum, so in total, we can accept 560 stacks of $e^{+}$from the third stage corresponding to $3.8(0.4) \times$ $10^{8} e^{+}$. We are planning to extend this process to trap even more positrons.

With the knowledge that the LINAC based positron source provides $1.54(0.06) \times 10^{5} e^{+}$per pulse at a frequency of $200 \mathrm{~Hz}$, the current overall efficiency is $2.3 \%$.

\section{Conclusion}

We succeeded in trapping $3.8(0.4) \times 10^{8} e^{+}$in $560 \mathrm{~s}$, which is a first important step for the development of the GBAR experiment. Improvements will be made in the coming months to reach our next step of $10^{10}$ positrons in a few hours, after which the goal will be to decrease this accumulation time, by increasing the $e^{+}$flux at the entrance, and by improving efficiencies at the interfaces of the system.

\section{Acknowledgments}

This research was partially supported by CNES under convention No. 5100017115, the French National Research Agency (grant ANR-14-CE33-0008), the Swiss National Science Foundation (grant 20FL21_173597) and the ETH Zurich (grant ETH-46 17-1).

\section{References}

[1] G. Chardin, et al. (GBAR Collaboration), "Proposal to Measure the Gravitational Behaviour of Antihydrogen at Rest", CERN-SPSC-2011-029, SPSC-P-342, 2011.

[2] P. Comini, P.-A Hervieux, F. Biraben Hyperfine Interact. 228, 159 (2014).

[3] J. Walz, T.W. Hänsch, Gen. Relativ. Gravitat. 36, 561 (2004).

[4] W. Oelert, et al. (ELENA Collaboration), Acta Phys. Pol. B 46, 181 (2015).

[5] P. Crivelli, U. Gendotti, A. Rubbia, L. Liszkay, P. Perez, C. Corbel, Phys. Rev. A 81, 052703 (2010).

[6] J.R. Danielson, D.H.E. Dubin, R.G. Greaves, C.M. Surko, Rev. Mod. Phys. 87, 247 (2015).

[7] A.M.M. Leite, Ph.D. Thesis, Paris-Saclay, 2017.

[8] P. Grandemange, Ph.D. Thesis, Université Paris Sud - Paris XI, 2013.

[9] L.V. Jørgensen (ATHENA Collaboration), Phys. Rev. Lett. 95, 025002 (2005).

[10] D.W. Fitzakerley (ATRAP Collaboration), J. Phys. B 49, 064001 (2016). 